\title{
Influence of ezetimibe on selected parameters of oxidative stress in rat liver subjected to ischemia/reperfusion
}

\author{
Małgorzata Trocha, Anna Merwid-Ląd, Ewa Chlebda, Tomasz Sozański, Małgorzata Pieśniewska, \\ Halina Gliniak, Adam Szeląg
}

Department of Pharmacology, Wroclaw Medical University, Poland

Submitted: 6 October 2011

Accepted: 1 April 2012

Arch Med Sci 2014; 10, 4: 817-824

DOI: 10.5114 /aoms.2013.38087

Copyright (c) 2014 Termedia \& Banach

\section{Abstract}

Introduction: Ischemia/reperfusion (I/R) is considered to be one of the main causes of liver damage after transplantation. The authors evaluated the effect of ezetimibe on selected oxidative stress parameters in ischemic/reperfused $(\mathrm{I} / \mathrm{R})$ rat liver.

Material and methods: Rats were administered ezetimibe ( $5 \mathrm{mg} / \mathrm{kg}$ ) (groups $\mathrm{E}$ and $E-I / R$ ) or saline solution (groups $C$ and $C-I / R$ ) intragastrically for 21 days. Livers of animals in groups $\mathrm{C}-\mathrm{I} / \mathrm{R}$ and $\mathrm{E}-\mathrm{I} / \mathrm{R}$ were subjected to $60 \mathrm{~min}$ of partial ischemia (left lateral and median lobes) followed by $4 \mathrm{~h}$ of reperfusion. Alanine and asparagine aminotransferase (ALT, AST) activity was determined in blood before I/R and during reperfusion (at 15 and $240 \mathrm{~min}$ ). After the reperfusion period, malondialdehyde (MDA), superoxide dismutase (SOD), glutathione (GSH) and glutathione peroxidase (GPX) were determined in liver homogenates using colorimetric methods.

Results: Ezetimibe caused a significant increase in GSH level in groups subjected to I/R $(\mathrm{E}-\mathrm{I} / \mathrm{R}(99.91 \pm 9.01)$ vs. C-I/R $(90.51 \pm 8.87), p<0.05)$. Additionally, under $I / R$ the decrease of GPx activity in the drug-treated group was lower compared to the non-treated group $(\mathrm{E}-\mathrm{I} / \mathrm{R}(3.88 \pm 1.11)$ Vs. $\mathrm{E}(5.31 \pm 1.83), p=0.076)$. Neither ezetimibe nor I/R affected SOD or MDA levels. I/R produced a significant increase in aminotransferase levels (ALT240-0: C-I/R (42.23 \pm 43.56$)$ vs. C (9.75 $\pm 11.09)$, and $\mathrm{E}-\mathrm{I} / \mathrm{R}(39.85 \pm 26.53)$ vs. $\mathrm{E}(4.38 \pm 1.36), p<0.05$ in both cases; AST 240-0: $\mathrm{E}-\mathrm{I} / \mathrm{R}(53.87 \pm 17.23)$ vs. $\mathrm{E}(24.10 \pm 9.66), p<0.05)$ but no effect of ezetimibe on those enzymes was found.

Conclusions: Ezetimibe demonstrates antioxidant properties in rat livers subjected to I/R. However, neither a hepatoprotective nor a hepatotoxic effect of ezetimibe was demonstrated, regardless of I/R.

Key words: ischemia/reperfusion, liver, rat, oxidative stress.

\section{Introduction}

Ischemia/reperfusion (I/R) is considered to be the main cause of cellular damage of various organs. The process occurs, among others, during a procedure of liver transplantation that has become a method of choice in the end-stage diseases of that organ [1]. Before implantation, liver damage occurs at two stages. Initially the injury is caused by ischemia. Further damage is caused by reperfusion of the organ. Pathogenesis of those lesions is very complex. Ischemia/reperfusion injury involves an early acute phase (3-6 h
Correspondence author: Małgorzata Trocha MD, PhD Department of Pharmacology Wroclaw Medical University 2 Mikulicz-Radecki St 50-345 Wroclaw, Poland Phone: +4871784 1442 Fax: +48717840094 E-mail: malgorzata.trocha@ gmail.com 
post reperfusion), involving generation of free radicals and nitric oxide, and activation of T-cells and Kupffer cells. That is followed by a subacute phase (18-24 $\mathrm{h}$ post reperfusion) characterized by neutrophil infiltration leading to continuous oxidant, cytokine, and chemokine production [2, 3].

During I/R-evoked oxidative stress, generation of reactive oxygen species (ROS) may exceed the capacity of endogenous redox degrading systems [2]. Malondialdehyde (MDA) is widely used as an indicator of oxidative damage. Non-enzymatic (e.g. glutathione (GSH)) and enzymatic (e.g. superoxide dismutase (SOD), and glutathione peroxidase (GPX)) antioxidants are involved in minimization of damaging effects of ROS on the liver [2, 4, 5]. Superoxide dismutase converts superoxide radicals to hydrogen peroxide $\left(\mathrm{H}_{2} \mathrm{O}_{2}\right)$ and oxygen [2]. Subsequently $\mathrm{H}_{2} \mathrm{O}_{2}$ becomes reduced by a selenium-containing enzyme - GPx - to water [6].

If the extent of damage in the liver preserved for transplantation largely depends on oxidative stress in liver cells, the view that drugs of antioxidant activity should be hepatoprotective seems justified. Ezetimibe belongs to a new class of 2-azetidinones - selective inhibitors of cholesterol absorption at the brush border of the intestine [7]. The drug interacts with the sterol transporter Niemann-Pick C1like protein (NPC1L1) [8]. The drug has been recently used in many potential liver donors.

Liver transplantation has presently become a procedure of choice in treatment of chronic and acute liver failure. In the USA over 3000 liver transplantations are performed annually [9]. Hyperlipidemia is a common metabolic disorder in the world population. It is estimated that in the UK two thirds of the population present an increased total cholesterol level [10], and only about $10 \%$ reaches the therapeutic goal for LDL level [11]. That requires treatment with high doses of statins in monotherapy or combined therapy with ezetimibe. A metaanalysis of 14 clinical trials revealed higher efficacy of combined therapy with simvastatin and ezetimibe compared to monotherapy with rosuvastatin [12]. Ezetimibe is characterized by good tolerance and low incidence of adverse effects [13]. The drug has become an increasingly popular therapeutic option for statin-resistant patients, as well as for statin-intolerant patients $[14,15]$. The choice of ezetimibe for our experiment was also based on data from experimental $[16,17]$ and clinical studies $[18,19]$ suggesting antioxidant properties of this agent.

Contrary to statins, pleiotropic effects of ezetimibe are still not well known and results of some works are contradictory [20-22]. Some properties of selected substances may appear only in stressful conditions such as I/R. The aim of this study was to evaluate antioxidative and hepatoprotective properties of ezetimibe that could be observed in rat liver subjected to I/R.

\section{Material and methods}

\section{Animals}

Wistar male rats obtained from the Animal Laboratory of the Department of Pathological Anatomy, Medical University of Wroclaw, were used for experiments. Animals were housed individually in chambers with a $12: 12 \mathrm{~h}$ light-dark cycle, temperature maintained at $21-23^{\circ} \mathrm{C}$. Before the experiment animals had free access to standard food and water. The experiment was approved by the $1^{\text {st }}$ Local Ethics Commission for Experiments on Animals in Wroclaw.

\section{Experimental protocol}

After adaptation, rats were randomly divided into four groups. Rats in groups C $(n=8)$ and $\mathrm{E}(n=8)$ were not exposed to I/R conditions. Rats in groups $\mathrm{C}-\mathrm{I} / \mathrm{R}(n=9)$ and E-I/R $(n=8)$ were exposed to 60 min of warm ischemia followed by $4 \mathrm{~h}$ of reperfusion. Rats in groups E and E-I/R were treated with intragastric ezetimibe, once daily for 21 days. Based on available literature data concerning efficacy of ezetimibe, the dose of $5 \mathrm{mg} / \mathrm{kg}$ b.w. was chosen for this experiment [23-26]. After 3 weeks of drug administration, blood samples $(0.8 \mathrm{ml})$ were collected from the tail vein to determine initial activities of alanine and asparagine aminotransferase (ALTO, ASTO). Subsequently, rats were anesthetized with intramuscular injection of ketamine $(7 \mathrm{mg} / \mathrm{kg})$ and medetomidine $(0.1 \mathrm{mg} / \mathrm{kg})$, and midline laparotomy was performed. In groups C-I/R and E-I/R $70 \%$ of liver ischemia (left lateral and median lobes) was achieved by occlusion of branches of the portal vein and of hepatic artery using a microvascular clip. Heparin $(200 \mathrm{U} / \mathrm{kg}$ ) was administered to rats to prevent blood coagulation. After $60 \mathrm{~min}$ of ischemia, the clip was removed to allow reperfusion for $4 \mathrm{~h}$. The abdomen was subsequently closed and rats were observed during reperfusion. At 15 and 240 min of reperfusion blood samples $(0.5 \mathrm{ml})$ were obtained from the tail vein, to determine aminotransferase activity (ALT15, ALT240 and AST15, AST240).

In groups $E$ and $C$ animals were anesthetized in the same way as in ischemic groups. Following midline laparotomy branches of the portal vein and hepatic artery were isolated but not occluded. After 60 min the abdomen was closed and rats were observed for $4 \mathrm{~h}$. Blood samples were obtained at the same time points as in the case of ischemic groups. After $4 \mathrm{~h}$ the left lateral and median lobes of livers were isolated to be compared with the same lobes obtained from ischemic livers.

Upon completion of the reperfusion, livers were isolated, weighed and homogenized on ice with lysis 
buffer (140 mM NaCl, 10 mM EDTA, 10\% glycerol, 1\% NP40, 20 mM Tris base, pH 7.5). Homogenized tissue was subsequently centrifuged at $14000 \mathrm{rpm}$ for $25 \mathrm{~min}$ at $4^{\circ} \mathrm{C}$ and supernatants were collected [27].

\section{Oxidative stress parameters and biochemical analyses}

The MDA, SOD, GPx and GSH were estimated in liver homogenates using colorimetric methods (spectrophotometer MARCEL S350 PRO). The GPX and SOD activities were assayed using the RANSEL and RANSOD kit, respectively (Randox Laboratories, Crumlin, UK). MDA and GSH concentrations were determined with the BIOXYTECH-MDA-586 and BIOXYTECH GSH-400 kit, respectively (OxisResearch, USA), according to the manufacturer's instructions.

Serum activities of ALT and AST and protein concentration in homogenates were assayed using a commercial enzymatic method in a certified laboratory. The SOD and GPx activities were expressed per milligram of protein. Activities of ALT and AST were expressed per gram of liver.

\section{Substances}

Ezetimibe (Ezetrol - tabl. $10 \mathrm{mg}$; MSD, UK), heparin (Heparinum WZF - amp. 25000 U/5 ml, Polfa Warszawa, Poland), ketamine hydrochloride

\section{A}

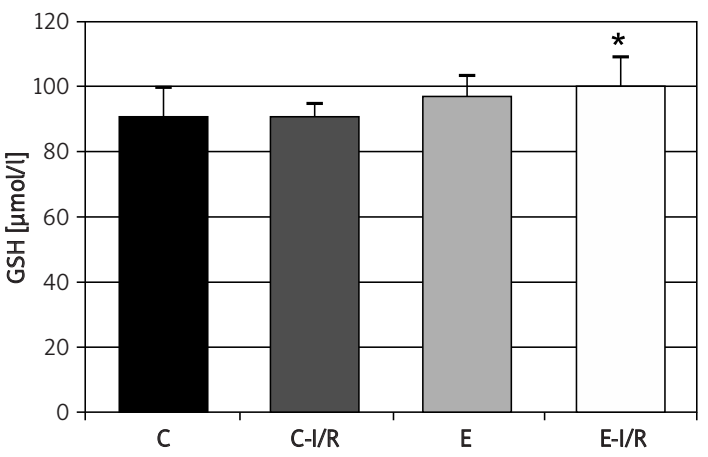

C

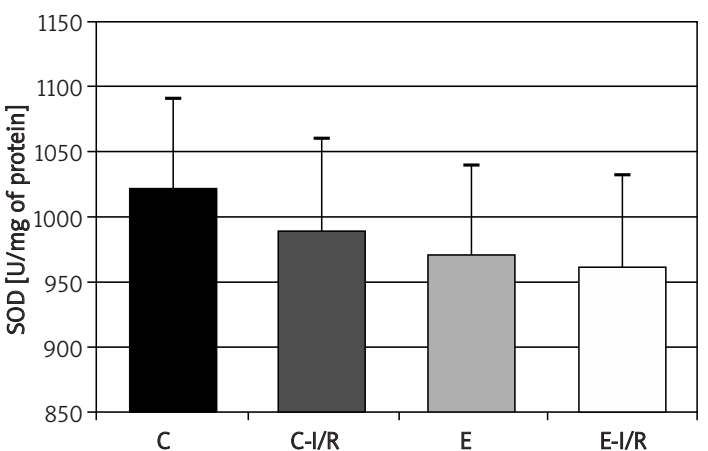

(Bioketan, Vetoquinol Biowet, Poland), medetomidine hydrochloride (Domitor, amp. $1 \mathrm{mg} / \mathrm{ml}$, Orion Pharma, Finland), $0.9 \%$ sodium chloride solution (Polpharma S.A., Poland), and Ringer solution (Polfa Lublin S.A., Poland) were used in these studies.

\section{Statistical analysis}

Data were expressed as mean values \pm SD. Statistical analysis of the drug and I/R effect on levels of oxidative stress parameters were performed using two-way analysis of variance (ANOVA). Statistical analysis of the effect of the drug and the time of reperfusion on ALT and AST levels was performed using MANOVA with repetitions. Contrast analysis was used for specific comparisons. Hypotheses were considered positively verified if $p<0.05$.

\section{Results}

The GSH levels were comparable in both nontreated groups (C-I/R vs. C, $p=N S$ ). In ezetimibetreated groups values of GSH were greater compared to non-treated groups, and in groups subjected to I/R the difference was significant (E-I/R vs. C-I/R, $p<0.05$ ) (Figure $1 \mathrm{~A}$ ).

The GPx activity was greater in non-I/R-exposed livers. Differences were significant in non-treated

B

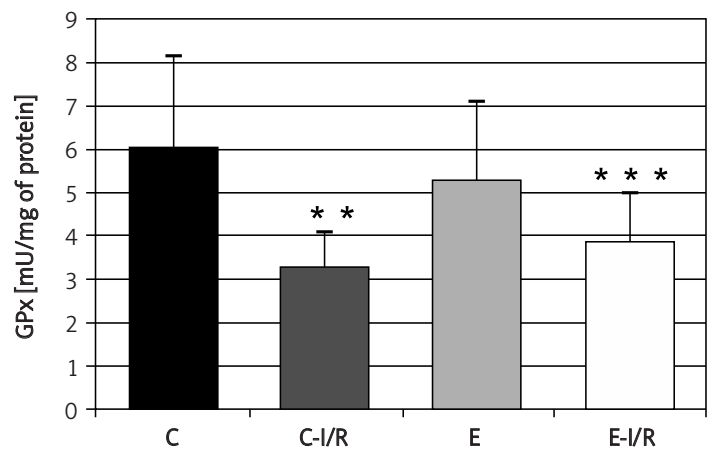

D

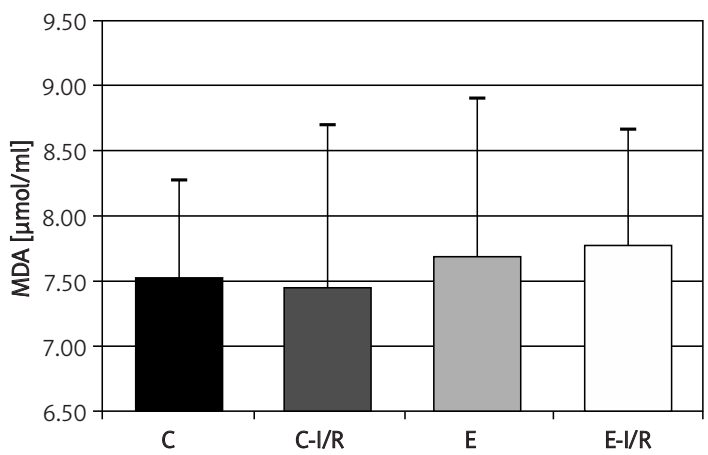

Figure 1. Influence of I/R and ezetimibe treatment on GSH (A), GPx (B), SOD (C), and MDA (D) levels. Values are presented as mean $\pm S D$. Group $C$ - not treated and not subjected to I/R; group $C-I / R$ - not treated and subjected to I/R; group E - ezetimibe-treated and not subjected to I/R; group E - ezetimibe-treated and subjected to I/R ${ }^{*} p<0.05(E-1 / R$ vs. $C-1 / R),{ }^{* *} p<0.005$ (C-I/R vs. C), ${ }^{* * *} p=0.076$ (E-I/R vs. E) 
animals (C-I/R vs. C, $p<0.005)$. In ezetimibe-treated animals the GPx decrease caused by I/R was of borderline significance (E-I/R vs. E, $p=0.076$ ) (Figure $1 \mathrm{~B})$.

The SOD activity and MDA level were similar in all groups of animals regardless of drug and $I / R$ exposure. I/R produced only an insignificant decrease in SOD activity ( $p=$ NS in both comparisons) (Figures $1 \mathrm{C}, 1 \mathrm{D}$ ).

After 3 weeks of administration ezetimibe did not alter ALT or AST activities and no significant differences between non-treated and ezetimibe-treated groups were found (C vs. E, $p=N S$ ) (Figures 2 $A$ and $2 \mathrm{~B}$ ).

The study showed a significant influence of liver I/R on AST and ALT activities. At $15 \mathrm{~min}$ and 240 min of reperfusion I/R produced a significant increase in ALT activity in ezetimibe-treated and non-treated groups (C-I/R vs. C and E-I/R vs. E, $p<0.05$ at both points of time in both cases) (Figure $2 \mathrm{~A}$ ). In the case of AST activity the rise of the value was not so pronounced. I/R caused a significant increase in AST level only in the drug-treated group (E-I/R vs. E, $p<0.05$ in 15 and $240 \mathrm{~min}$ ) (Figure $2 \mathrm{~B}$ ). The increase in ALT activity between 0 and 240 min of reperfusion was significantly higher in I/R-subject groups compared to the groups not exposed to I/R (C-I/R vs. C and E-I/R vs. E, $p<0.05$ in both cases) (Figure $1 \mathrm{~A}$ ). In the case of AST, a significant difference was found only between both drug-treated groups (E-I/R vs. E, $p<0.05$ ) (Figure $2 \mathrm{~B}$ ).

Under I/R conditions in ezetimibe-treated groups the level of aminotransferases was lower compared to non-treated groups, but the difference between $\mathrm{C}-\mathrm{I} / \mathrm{R}$ and $\mathrm{E}-\mathrm{I} / \mathrm{R}$ was not significant ( $p=\mathrm{NS}$ ). No significant increase of either liver enzyme was also observed between 0 and 240 min of reperfusion (E-I/R vs. C-I/R, $p=$ NS in the case of both enzymes) (Figure $2 \mathrm{~A}$ and $2 \mathrm{~B}$ ).

A

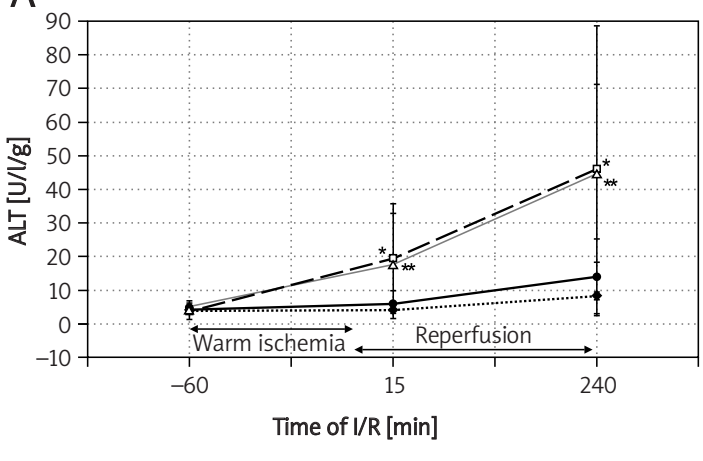

\section{Discussion}

In our study we have demonstrated that: (1) the effect of long-term administered ezetimibe on oxido-redox status was present in I/R-subjected groups - the GSH level was significantly higher in the ezetimibe-treated group compared to the nontreated group; (2) the influence of ezetimibe on GPx activity was not so evident as on GSH level and was present in the form of a less pronounced decrease under I/R conditions; (3) no hepatotoxic effect of ezetimibe was found - on the contrary, even a slightly positive effect on liver function in groups subjected to I/R was demonstrated.

In many papers, conditions including dyslipidemia, obesity, and diabetes mellitus are used as examples of aggravated oxidative stress. Pleiotropic activity of some drugs is examined in those conditions $[28,29]$. During a transplantation procedure oxidative stress associated with I/R injury is also observed [30, 31]. In our study, as in many other reports [32, 33], decreased activity of GPx indicated that this is a very sensitive enzyme. Other examined antioxidants (SOD, GSH) were not affected by I/R in our experiment. In various reports, however, SOD activity also remained unaffected, suggesting that the enzyme is a less sensitive predictor of oxidative stress [34, 35]. The level of MDA - one of the most popular indicators of oxidative stress [31, 36] - was also unchanged in I/R groups. That could be a result of too short time of ischemia or reperfusion. In those conditions liver injuries were not sufficiently pronounced. Probably, MDA or SOD and GSH levels should also be measured after 24-48 h, during the second phase of reperfusion. Another possibility to evoke more pronounced oxidative stress is to create in the experiment a group of rats with hyperlipidemia evoked by e.g. high cholesterol diet.

Antioxidative properties of many substances have been studied in various models of I/R. A large

B

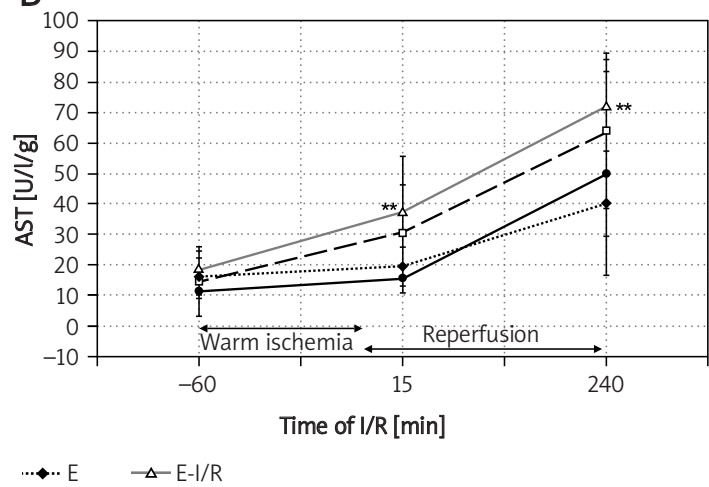

Figure 2. Effect of I/R and ezetimibe treatment on ALT (A) and AST (B) activities. Values are presented as mean \pm SD. Group C - not treated and not subjected to I/R; group C-I/R - not treated and subjected to I/R; group E - ezetimibe-treated and not subjected to I/R; group $\mathrm{E}$ - ezetimibe-treated and subjected to I/R ${ }^{*} p<0.05$ (C-I/R vs. C), ${ }^{* *} p<0.05$ (E-I/R vs. E) 
body of evidence concerns statins [37, 38]. Administration of rosuvastatin before spinal cord ischemia caused an increase in SOD and GPx activities along with a decrease of MDA level that was accompanied by decreased spinal cord necrosis [39]. Decreased MDA and free radical levels were also observed in an intestine I/R model, with a beneficial effect of L-arginine on those changes as well as on increased leukocyte adherence in ischemic tissues [40]. Combination of L-arginine with a wellknown antioxidant - $\alpha$-tocopherol - markedly decreased the MDA level and severity of acute tubular necrosis in a rat model of kidney I/R [41]. Pleiotropic properties of ezetimibe have been reported by other authors, but they still remain not well documented [20-22]. Effects of ezetimibe including reduction of ADMA level, increase in eNOS mRNA expression and reduction in liver lipids were demonstrated [42]. The drug also decreased platelet aggregation in patients with hyperlipidemia [43], along with weight loss, lowered plasma hs-CRP and IL- 6 levels, and TNF- $\alpha$ concentration in obese and diabetic patients [44], and with atorvastatin improved endothelium-dependent vasodilatation and blood flow [20]. Ezetimibe as a therapy "complementary" to statins remarkably reduced a circulating oxidative stress marker - derivatives of reactive oxidative metabolites (dROMs) - in patients with hypercholesterolemia, probably by decreasing the tendency to peroxidation of LDL and reducing circulating ox-LDL level [45]. Ezetimibe alone also reduced dROMs levels, but only in patients with "high oxidative stress" at baseline [46]. In patients with non-diabetic chronic kidney disease (CKD) ezetimibe enhanced proteinuria-lowering effects of pitavastatin [47]. That drug decreased the level of 8-hydroxydeoxyguanosine - a marker of oxidative stress - so its nephroprotective activity may be associated with its antioxidative properties [48]. Monotherapy with ezetimibe decreased MDA-LDL and total LDL levels in patients with hyperlipidemia and chronic heart disease, ameliorating vascular function by decreasing oxidative stress. Based on this clinical study ezetimibe seems to be a promising agent not only as a second-choice lipid-lowering drug, but also - considering its pleiotropic effects on blood vessels - as a main agent for that group of patients [18]. But in other reports ezetimibe exerted a rather minor effect on atherogenic dyslipidemia. Addition of ezetimibe to simvastatin in patients with heterozygous familial hypercholesterolemia did not cause a change in mean thickness of carotid intima-media [49]. Ezetimibe-associated improvement of antioxidant state in the liver under I/R conditions observed in our experiment may suggest higher transplant survival due to e.g. potential improvement of vasculature function. However, it must be emphasized that some bene- ficial changes in oxido-redox parameters observed in experimental studies on animals are not compatible with clinical observations in humans.

In our experiment, ezetimibe administered for an extended period of time to rats not subjected to I/R did not change oxidative stress parameters in the liver. In livers subjected to I/R the influence of ezetimibe was reflected by an increase of GSH level. Another parameter that could suggest a positive impact of ezetimibe on oxidative stress is GPX, because in I/R conditions the decrease of activity of that enzyme is not equally pronounced compared to the non-treated group. Those observations may suggest better protection by GSH and GPx in those livers and improved antioxidant defense. But for better understanding of antioxidative properties of ezetimibe in those conditions future studies are required. A lot of studies on ezetimibe action have been carried out on animals or patients with oxidative stress evoked by dyslipidemia [18, 42, 43, 45, 49]. As we know, many people, including potential liver donors, suffer from hyperlipidemia and liver steatosis; therefore the results of an experiment with oxidative stress resulting from I/R along with hyperlipidemia could also be very interesting and may have clinical meaning. There is high probability that oxido-redox disorders will be more pronounced because the pathogenesis of this state will result from two simultaneous causes. Similarly, the effect of ezetimibe will be related not only to potential pleiotropic properties, but also to its hypolipemic action. Regardless of $\mathrm{I} / \mathrm{R}$, reduction of the lipid concentration will improve oxido-redox status.

Short-term administration to rodents of such compounds as dihydrolipoyl histidinate zinc complex [50], vitamin E succinate [51], N-acetylcysteine [52] or (-)-epigallocatechin gallate [53] revealed their protective or antioxidant effects on liver subjected to ischemia and reperfusion. Therefore an experiment designed in a way that a single dose of a drug is administered just before the I/R procedure would be a valuable supplementation of the studies on ezetimibe-evoked pleiotropic effects.

It is known that liver decays during the time of ischemia and reperfusion. Aminotransferases are hepatocellular enzymes the activity of which increases during hepatocyte damage such as observed in I/R [30, 31]. As expected, in our experiment we observed the most pronounced liver injury in groups exposed to I/R. Differences in enzymatic activities between I/R-exposed and non-I/ R-exposed livers appeared at time points 15 and 240 min of reperfusion.

The effect of ezetimibe treatment on liver injury is another interesting problem. The safety profile of the drug evaluated in many studies is satisfactory and the risk of liver damage is not increased - only a slight increase in activity of aminotrans 
ferases was observed [37, 54]. Of note, a few cases of serious liver damage - cholestatic and acute immune hepatitis - have been reported $[55,56]$. Ezetimibe-induced liver damage could be a result of expression of NPC1L1 on human liver cells [55], or synthesis of auto-antibodies directed against ezetimibe-metabolizing enzymes [57]. On the other hand, several recent studies have shown that this drug attenuated liver steatosis in experimental nonalcoholic fatty liver disease and could be a useful agent in treatment of that disorder [58, 59]. The drug could also be effective in treatment of hypercholesterolemia in liver recipients [60, 61].

Effects of a long-term administered drug on the liver may not be revealed until the I/R condition develops, e.g. during liver transplantation. Initially unaltered activity of liver enzymes may change, indicating worsening or improvement of liver function. Based on our results we believe that treatment of liver donors with ezetimibe does not impair liver function. Even more, in our experiment a slight beneficial effect of ezetimibe on ALT and AST activities was observed. After $4 \mathrm{~h}$ of reperfusion aminotransferase levels in non-treated groups were higher than in drug-treated groups but the difference was not significant. Usefulness of ezetimibe in nonalcoholic fatty liver disease [58, 59] and hyperlipidemia after liver transplantation [60, 61] as well a slight beneficial effect on aminotransferase activities in our experiment indicated that potential results of an experiment with a rat group with dietevoked hyperlipidemia may be extremely interesting. Protective action of ezetimibe would be related not only to potential antioxidative properties, but also to the main drug action reducing LDL and total cholesterol.

In conclusion, ezetimibe did not cause any noticeable protective changes in rat livers not subjected to $I / R$, because all parameters of oxidative stress and aminotransferase activities were comparable in both non-treated and drug-treated groups. However, results obtained under I/R conditions suggest that ezetimibe could have protective properties in liver under $I / R$, but those findings should be confirmed in subsequent studies. In our report the observed beneficial antioxidative effect of ezetimibe was based on changes of concentration of GSH and, to a lower extent, on activity of GPx. No hepatotoxic features of this drug were noted. On the contrary, even a slight protective effect on liver function under I/R may be suggested.

\section{Acknowledgments}

The study was financially supported by the statutory means of Wroclaw Medical University (ST-49). The study was supported by a research fellowship within the "Development Program of Wroclaw Medical University" funded from the European Social
Fund, Human Capital, National Cohesion Strategy (contract no. UDA-POKL.04.01.01-00-010/08-01). The authors declare no conflict of interest.

\section{References}

1. Cobreros A, Sainz L, Lasheras B, Cenarruzabeitia E. Hepatotoxicity of ethanol: protective effect of calcium channel blockers in isolated hepatocytes. Liver 1997; 17: 76-82.

2. Fan C, Zwacka RM, Engelhardt JF. Therapeutic approaches for ischemia/reperfusion injury in the liver. J Mol Med 1999; 77: 577-96.

3. Hines IN, Harada H, Flores S, Gao B, McCord JM, Grisham MB. Endothelial nitric oxide synthase protects the post-ischemic liver: potential interactions with superoxide. Biomed Pharmacother 2005; 59: 183-9.

4. Scandalios JG. Oxidative stress: molecular perception and transduction of signals triggering antioxidant gene defenses. Braz J Med Biol Res 2005; 38: 995-1014.

5. Alan C, Kocoglu H, Altintas R, Alici B, Ersay AR. Protective effect of decorin on acute ischaemia-reperfusion injury in the rat kidney. Arch Med Sci 2011; 2: 211-6.

6. Wassmann S, Wassmann K, Nickenig G. Modulation of oxidant and antioxidant enzyme expression and function in vascular cells. Hypertension 2004; 44: 381-6.

7. Sudhop T, von Bergmann K. Cholesterol absorption inhibitors for the treatment of hypercholesterolemia. Drugs 2002; 62: 2333-47.

8. Gracia-Cavlo M, Lisnock JM, Bull HG, et al. The target of ezetimibe is Niemann-Pick C 1-Like 1 (NPC1L1). Proc Natl Acad Sci USA 2005; 102: 8132-7.

9. Habior A, Rawa T, Krawczyk M, et al. Liver transplantation - evaluation requirements, indications and rules for qualification [Polish]. Med Science Rev Hepatologia Med J For Cont, 85-90.

10. Jessani S, Watson T, Cappuccio FP, et al. Prevention of cardiovascular disease in clinical practice: the Joint British Societies' (JBS 2) guidelines. J Hum Hypertens 2006; 20: 641-5.

11. Kawecka-Jaszcz K, Jankowski P, Pajak A. Determinants of appropriate lipid management in patients with ischaemic heart disease. Cracovian Program for Secondary Prevention of Ischaemic Heart Disease. Int J Cardiol 2003; 91: 15-23.

12. Pearson TA, Denke MA, McBride PE, Battisti WP, Brady WE, Palmisano J. A community-based, randomized trial of ezetimibe added to statin therapy to attain NCEP ATP III goals for LDL cholesterol in hypercholesterolemic patients: the ezetimibe add-on to statin for effectiveness (EASE) trial. Mayo Clin Proc 2005; 80: 587-95.

13. Pikto-Pietkiewicz W, Pasierski T. Ezetimibe - cholesterol absorption inhibitor [Polish]. Kardiol Pol 2006; 64: 1434-41.

14. Shekhar Pandey A, Bissonnette S, Boukas S, Rampakakis E, Sampalis JS. Effectiveness and tolerability of ezetimibe co-administered with statins versus statin dose-doubling in high-risk patients with persistent hyperlipidemia: The EZE(STAT)2 trial. Arch Med Sci 2011; 7: 767-75.

15. Rizzo M, Barylski M, Rizvi AA, Montalto G, Mikhailidis DP, Banach M. Combined dyslipidemia: should the focus be LDL cholesterol or atherogenic dyslipidemia? Curr Pharm Des 2013; 19: 3858-68.

16. Fukuda M, Nakamura T, Kataoka K, et al. Ezetimibe ameliorates cardiovascular complications and hepatic steatosis in obese and type 2 diabetic $\mathrm{db} / \mathrm{db}$ mice. J Pharmacol Exp Therap 2010; 335: 70-5. 
17. Nakagami H, Osako MK, Takami Y, et al. Vascular protective effects of ezetimibe in apoE-deficient mice. Atherosclerosis 2009; 203: 51-8.

18. Kurobe $\mathrm{H}$, Aihara KI, Higashida $M$, et al. Ezetimibe monotherapy ameliorates vascular function in patients with hypercholesterolemia through decreasing oxidative stress. J Atheroscler Thromb 2011; 18: 1080-9.

19. Yamaoka-Tojo M, Tojo T, Takahira N, Masuda T, Izumi T. Ezetimibe and reactive oxygen species. Curr Vasc Pharmacol 2011; 9: 109-20.

20. Bulut D, Hanefeld C, Bulut-Streich N, Graf C, Mugge A, Spiecker M. Endothelial function in the forearm circulation of patients with the metabolic syndrome - effect of different lipid-lowering regimens. Cardiology 2005; 104: 176-80.

21. Landmesser U, Bahlmann F, Mueller M, et al. Simvastatin versus ezetimibe: pleiotropic and lipid-lowering effects on endothelial function in humans. Circulation 2005; 111: 2356-63.

22. Kalogirou M, Tsimihodimos V, Elisaf M. Pleiotropic effects of ezetimibe: do they really exist? Eur J Pharmacol 2010; 633: 62-70.

23. Cheng Y, Liu F, Wu J, Zhang Y, Nilsson A, Duan RD. Ezetimibe inhibits expression of acid sphingomyelinase in liver and intestine. Lipids 2009; 44: 897-906.

24. Oswald S, May K, Rosin J, Lütjohann D, Siegmund W. Synergistic influence of Abcb1 and Abcc2 on disposition and sterol lowering effects of ezetimibe in rats. J Pharm Sci 2010; 99: 422-9.

25. Hayashi H, Kanai S, Yamada Y, Tsushima M, Miyasaka K. Acute inhibition of lymphatic cholesterol transport in rat intestine by SCH 58053. J Atheroscler Thromb 2006; 13: 240-6.

26. Oswald S, Westrup S, Grube M, Kroemer HK, Weitschies W, Siegmund W. Disposition and sterol-lowering effect of ezetimibe in multidrug resistance-associated protein 2-deficient rats. J Pharmacol Exp Ther 2006; 318: 1293-9.

27. Morales Al, Vicente-Sanchez C, Jerkic M, et al. Effect of quercetin on metallothionein, nitric oxide synthase and cyclooxygenase-2 expression on experimental chronic cadmium nephrotoxicity in rats. Toxicol Appl Pharmacol 2006; 210: 128-35.

28. Turfaner N, Uzun $\mathrm{H}$, Balci $\mathrm{H}$, et al. Ezetimibe therapy and its influence on oxidative stress and fibrinolytic activity. South Med J 2010; 103: 428-33.

29. Nomura M, Ishii H, Kawakami A, Yoshida M. Inhibition of hepatic Niemann-Pick C1-like 1 improves hepatic insulin resistance. Am J Physiol Endocrinol Metab 2009; 297: E1030-8.

30. Montalvo-Javé EE, García-Puig MA, Escalante-Tattersfield T, Peña-Sánchez J, Vázquez-Meza H, Ortega-Salgado JA. Biochemical analysis and lipid peroxidation in liver ischemic preconditioning. Cir Cir 2011; 79: 132-40.

31. Wu C, Wang P, Rao J, et al. Triptolide alleviates hepatic ischemia/reperfusion injury by attenuating oxidative stress and inhibiting NF-kappaB activity in mice. J Surg Res 2011; 166: e205-13.

32. Somuncu S, Cakmak M, Dikmen G, Akman H, Kaya M. Ischemia-reperfusion injury of rabbit ovary and protective effect of trapidil: an experimental study. Pediatr Surg Int 2008; 24: 315-8.

33. Turgut F, Bayrak O, Catal F, et al. Antioxidant and protective effects of silymarin on ischemia and reperfusion injury in the kidney tissues of rats. Int Urol Nephrol 2008; 40: 453-60.

34. Ceylan A, Karasu C, Aktan F, Güven C, Can B, Ozansoy G. Effects of simvastatin treatment on oxidant/antioxidant state and ultrastructure of diabetic rat myocardium. Gen Physiol Biophys 2003; 22: 535-47.

35. Ozansoy G, Akin B, Aktan F, Karasu C. Short-term gemfibrozil treatment reverses lipid profile and peroxidation but does not alter blood glucose and tissue antioxidant enzymes in chronically diabetic rats. Mol Cell Biochem 2001; 216: 59-63.

36. Xue F, Zhang JJ, Xu LM, Zhang C, Xia Q. Protective effects of HGF-MSP chimer (metron factor-1) on liver ischemiareperfusion injury in rat model. J Dig Dis 2010; 11: 299-305.

37. Jankowski P, Loster M, Kawecka-Jaszcz K. Ezetimibe: new perspectives in lipid lowering treatment. Cardiol J 2007; 14: 232-7.

38. Efrati S, Averbukh M, Dishy V, Faygenzo M, Friedensohn L, Golik A. The effect of simvastatin, ezetimibe and their combination on the lipid profile, arterial stiffness and inflammatory markers. Eur J Clin Pharmacol 2007; 63: 113-21.

39. Ucak A, Onan B, Güler A, et al. Rosuvastatin, a new generation 3-hydroxy-3-methylglutaryl coenzyme a reductase inhibitor, reduces ischemia/reperfusion-induced spinal cord tissue injury in rats. Ann Vasc Surg 2011; 25: 686-95.

40. Krauss $H$, Sosnowski P, Biczysko M, et al. Effects of $L$ arginine and NG-nitro L-arginine methyl ester (L-NAME) on ischemia/reperfusion injury of skeletal muscle, small and large intestines. Chin J Physiol 2011; 54: 7-18.

41. Shokeir AA, Barakat N, Hussein AA, Awadalla A, Abdel-Aziz $A$, Abo-Elenin $\mathrm{H}$. Role of combination of $\mathrm{L}$-arginine and alpha-tocopherol in renal transplantation ischaemia/ reperfusion injury: a randomized controlled experimental study in a rat model. BJU Int 2011; 108: 612-8.

42. Stapleton PA, Goodwill AG, James ME, Brock RW, Frisbee JC. Hypercholesterolemia and microvascular dysfunction: interventional strategies. J Inflam 2010; 7: 1-10.

43. Hussein O, Minasian L, Itzkovich Y, Shestatski K, Solomon L, Zidan J. Ezetimibe's effect on platelet aggregation and LDL tendency to peroxidation in hypercholesterolemia as monotherapy or in addition to simvastatin. Br J Clin Pharmacol 2007; 65: 637-45.

44. Chan DC, Watts GF, Gan SK, Ooi EMM, Hugh P, Barrett R. Effect of ezetimibe on hepatic fat, inflammatory markers, and apolipoprotein B-100 kinetics in insulin-resistant obese subjects on a weight loss diet. Diabetes Care 2010; 33: 1134-9.

45. Yamaoka-Tojo M, Tojo T, Kosugi R, et al. Effects of ezetimibe add-on therapy for high-risk patients with dyslipidemia. Lipid Health Dis doi: 10.1186/1476-511X8-41.

46. Kostapanos MS, Spyrou AT, Tellis CC, et al. Ezetimibe treatment lowers indicators of oxidative stress in hypercholesterolemia subjects with high oxidative stress. Lipids 2011; 46: 341-8.

47. Nakamura T, Sato E, Fujiwara N, et al. Co-administration of ezetimibe enhances proteinuria-lowering effects of pitavastatin in chronic kidney disease patients partly via a cholesterol-independent manner Pharmacol Res 2010 61: 58-61.

48. Nakamura T, Sato E, Fujiwara N, et al. Ezetimibe decreases serum levels of asymmetric dimethylarginine (ADMA) and ameliorates renal injury in non-diabetic chronic kidney disease patients in a cholesterol-independent manner. Pharmacol Res 2009; 60: 525-8.

49. Rizzo M, Rini GB. Ezetimibe, cardiovascular risk and atherogenic dyslipidaemia. Arch Med Sci 2011; 7: 5-7.

50. Masuda T, Iwashita Y, Hagiwara S, et al. Dihydrolipoyl histidinate zinc complex, a new antioxidant, attenuates 
hepatic ischemia-reperfusion injury in rats. Gastroenterol Hepatol 2011; 26: 1652-8.

51. Evans ZP, Mandavilli BS, Ellett JD, et al. Vitamin E succinate enhances steatotic liver energy status and prevents oxidative damage following ischemia/reperfusion. Transplant Proc 2009; 41: 4094-8.

52. Castro AP, Castro Junior MA, Lauz S, Facin E, Simões Mde J, Fagundes DJ. The role of $\mathrm{N}$-acetyl-cysteine in the lung remote injury after hepatic ischemia and reperfusion in rabbits. Acta Cir Bras 2012; 27: 49-55.

53. Fiorini RN, Donovan JL, Rodwell D, et al. Short-term administration of (-)-epigallocatechin gallate reduces hepatic steatosis and protects against warm hepatic ischemia/reperfusion injury in steatotic mice. Liver Transpl 2005; 11: 298-308.

54. Castellote J, Ariza J, Rota R, GirbauA, Xiol X. Serious druginduced disease secondary to ezetimibe. World J Gastroenterol 2008; 14: 5098-9.

55. Liu Q, Tobias H, Petrovic LM. Drug-induced liver injury associated with ezetimibe therapy. Dig Dis Sci 2007; 52: 602-5.

56. Stolk MF, Becx MC, Kuypers KC, Seldenrijk CA. Severe hepatic side effects of ezetimibe. Clin Gastroenterol Hepatol 2006; 4: 908-11.

57. Obermayer-Straub P, Strassburg CP, Manns MP. Target proteins in human autoimmunity: cytochromes P450 and UDP-glucuronosyltransferases. Can J Gastroenterol 2000; 14: 429-39.

58. Park H, Shima T, Yamaguchi K, et al. Efficacy of long-term ezetimibe therapy in patients with nonalcoholic fatty liver disease. J Gastroenterol 2011; 46: 101-7.

59. Ogawa T, Fujii H, Yoshizato K, Kawada N. A human-type nonalcoholic steatohepatitis model with advanced fibrosis in rabbits. Am J Pathol 2010; 177: 153-65.

60. Almutairi F, Peterson TC, Molinari M, Walsh MJ, Alwayn I, Peltekian KM. Safety and effectiveness of ezetimibe in liver transplant recipients with hypercholesterolemia. Liver Transpl 2009; 15: 504-8.

61. Savvidaki E, Koukoulaki M, Benou A, et al. Ezetimibe is effective in the treatment of persistent hyperlipidemia of renal allograft recipients. Clin Nephrol 2011; 75: 107-12. 